\title{
СТЕГАНОГРАФИЧЕСКОЕ ВСТРАИВАНИЕ ДОПОЛНИТЕЛЬНЫХ ДАННЫХ В СНИМКИ ДИСТАНЦИОННОГО ЗОНДИРОВАНИЯ ЗЕМЛИ С ПОМОЩЬЮ МЕТОДА QІМ С ПЕРЕМЕННЫМ ШАГОМ КВАНТОВАНИЯ В ЧАСТОТНОЙ ОБЛАСТИ
}

\section{Евсютин Олег Олегович',} eoo@keva.tusur.ru

\author{
Кокурина Анна Сергеевна', \\ annakokurina94@yandex.ru
}

\author{
Мещеряков Роман Валерьевич², \\ mrv@ipu.ru
' Томский государственный университет систем управления и радиоэлектроники, Россия, 634050, г. Томск, пр. Ленина, 40.
2 Институт проблем управления им. В.А. Трапезникова Российской академии наук, Россия, 117997, г. Москва, ул. Профсоюзная, 65.

\begin{abstract}
Актуальность. Одно из направлений обработки цифровых изображений, в том числе снимков дистанционного зондирования Земли, связано со встраиванием в них дополнительной информации различного назначения. Встраивание дополнительной информации в цифровое изображение приводит к искажению естественной модели цифрового изображения, а также к возможному возникновению визуальных артефактов. В случае снимков дистанционного зондирования Земли такие искажения могут повлечь, например, искажение границ объектов, в результате чего дальнейший анализ снимков приведёт к некорректным результатам. Поэтому актуальными являются исследования, направленные на поиск новых способов уменьшения искажений, вызванных встраиванием дополнительной информации.

Цель: повышение качества стеганографического встраивания информации в коэффициенты дискретного косинусного преобразования снимков дистанционного зондирования Земли за счёт разработки улучшенного алгоритма встраивания, основанного на методе QIM и обеспечивающего корректировку искажений естественной модели изображения в частотной области.

объекты: алгоритмы стеганографического встраивания информации в коэффициенты дискретного косинусного преобразования цифровых изображений.

Методы: стеганографический метод QIM, методы математической статистики, вычислительные эксперименты.

Результаты. Предлагается новый подход к минимизации искажений естественной модели цифрового изображения в области дискретного косинусного преобразования, основанный на изменении шага квантования в зависимости от локальных характеристик изображения в частотной области. Результатом работы является улучшенный алгоритм встраивания информации в область дискретного косинусного преобразования снимков дистанционного зондирования Земли, объединяющий данный подход с подходом к обеспечению безошибочного извлечения встроенной информации, предложенным авторами ранее. Результаты экспериментов показывают, что разработанный алгоритм наряду с обеспечением безошибочности извлечения позволяет существенно уменьшить искажения, вносимые в частотные коэффициенты, и обеспечить статистическую неразличимость исходных изображений и стегоизображений в 75 \% случаев.
\end{abstract}

\section{Ключевые слова:}

Цифровые изображения, стеганография, дискретное косинусное преобразование, QIM, дистанционное зондирование Земли.

\section{Введение}

Использование снимков дистанционного зондирования Земли (ДЗЗ) актуально в разных сферах деятельности человека, например, для создания карт местности, мониторинга состояния поверхности Земли, поиска месторождений полезных ископаемых, тектонических исследований, оценки состояния сельскохозяйственных угодий и лесных покровов и т. д. Данные ДЗЗ - это аэрокосмические снимки, т. е. цифровые изображения, следовательно, для их обработки и анализа применяются различные методы обработки и анализа цифровых изображений, такие как сегментация [1], фильтрация и выделение контуров [2], распознавание объектов [3] и другие [4]. Одним из перспективных направлений исследований в области обработки цифровых изображений, в частности снимков ДЗ3, является сокрытие дополнительной информации в них.
Встраивание информации различного назначения в данные ДЗЗ в настоящее время исследуется многими авторами. В большинстве случаев под сокрытием информации подразумевается встраивание цифровых водяных знаков (ЦВЗ) - информационных последовательностей небольшого объёма, заранее заданных или сгенерированных на основе содержимого контейнера. Обычно целью внедрения ЦВЗ в изображения является защита их подлинности и целостности. Например, в работе [5] представлена схема встраивания ЦВЗ в изображения ДЗ3 с применением векторного квантования. Авторы работы [6] также предлагают обеспечивать целостность снимков ДЗЗ с помощью встраивания ЦВЗ, используя для этого сдвиг гистограммы. Причём описанный ими подход является обратимым, т. е. позволяет полностью восстановить исходный снимок после извлечения встро- 
енной информации. В работе [7] представлен алгоритм генерации стойкого ЦВЗ для защиты гиперспектральных изображений д3З, обеспечивающий высокую устойчивость встроенного ЦВЗ к атакам прямого перебора ключа. Авторы статьи [8] акцентируют внимание на важности слепого обнаружения ЦВЗ и предлагают соответствующий подход.

Другим направлением сокрытия информации в цифровых объектах является стеганографическое встраивание. Цифровая стеганография предназначена для скрытой передачи информации внутри некоторых цифровых объектов. Она может использоваться либо для обеспечения конфиденциальности передаваемых в цифровых контейнерах данных, либо для хранения в цифровых контейнерах некоторого объёма информации, обычно большего, чем в случае ЦВЗ. Например, в статье [9] представлен алгоритм скрытия информации в кадрах видеофайлов, полученных со спутников, для безопасной передачи и архивирования конфиденциальных данных. В работе [10] предлагается алгоритм встраивания информации в гиперспектральные снимки ДЗ3, отличающийся высокой ёмкостью, причём встраивание защищаемой информации комбинируется со сжатием изображений-контейнеров. С помощью методов цифровой стеганографии также возможно скрывать в снимках ДЗ3 различную служебную информацию, связанную с происхождением снимка, с характеристиками зафиксированного участка поверхности Земли и т. д., тем самым уменьшая количество сопутствующих данных, требуемых при хранении и передаче снимка, и упрощая процедуру дальнейшей обработки.

Одним из основных требований к методам встраивания информации является возможность ее последующего безошибочного извлечения. Это особенно актуально для частотного встраивания, когда биты сообщения записываются в коэффициенты, полученные в результате применения к изображению-контейнеру некоторого частотного преобразования. В результате выполнения прямого и обратного частотного преобразования и последующего округления часть информации теряется, и восстановление встроенного сообщения в исходном виде не представляется возможным. Очевидно, что в случае сокрытия служебной информации в снимках Д3З безошибочность извлечения является обязательным критерием эффективности встраивания, поскольку потеря или изменение даже одного-двух битов могут повлечь искажение встроенных данных, что сделает дальнейшую работу с ними бессмысленной.

Ранее авторами настоящего исследования был предложен новый подход к обеспечению безошибочности, заключающийся в итеративном исправлении ошибок при извлечении информации, и получен алгоритм, основанный на методе модуляции индекса квантования (QIM) в области дискретного косинусного преобразования (ДКП), который обеспечивает безошибочность извлечения встроенной информации за счет предложенного подхода [11].
Другим важным требованием к стеганографическим методам является незаметность встраивания, поскольку сокрытие дополнительной информации в цифровом объекте-контейнере не должно оказывать существенного влияния на дальнейшие анализ и обработку контейнера. Во многих случаях внедрение дополнительной информации в элементы данных, составляющие цифровое изображение, нарушает естественный вид модели цифрового изображения. В результате изображения-контейнеры и стегоизображения становятся статистически различимы. Вид искажений зависит от операции встраивания.

Данная работа развивает исследование [11] и предлагает новый подход к минимизации искажений естественной модели цифрового изображения в области ДКП при встраивании по методу QIM, основанный на изменении шага квантования в зависимости от локальных характеристик изображения в частотной области.

Основным результатом работы является новый улучшенный алгоритм встраивания информации в область ДКП цифровых изображений, одновременно обеспечивающий безошибочность встраивания и статистическую неразличимость изображений-контейнеров и стегоизображений. Данный алгоритм может эффективно использоваться для внедрения дополнительной информации в снимки ДЗЗ.

Описание и обоснование решений, лежащих в основе разработанного алгоритма, представлено в следующих разделах данной статьи.

\section{Обеспечение безошибочного извлечения} информации, встроенной по методу QIM

Основная идея метода QIM [12] заключается в модуляции яркости пикселей или значений частотных коэффициентов в зависимости от значений встраиваемых битов. Существует большое количество вариаций классического метода QIM. Например, в работе [13] представлена основанная на QIM техника внедрения цифровых водяных знаков для трёхмерных сеток. Авторы работы [14] предлагают улучшенную версию QIM, обеспечивающую устойчивость к масштабированию и ряду других искажений. В [15] представлен вариант метода QIM для JPEG-изображений, направленный на повышение устойчивости к атакам, основанным на гистограммах. В статье [16] описан метод стеганографического встраивания информации в область ДВП для изображений в формате JPEG2000. Авторами работы [17] предлагается использовать двойное вложение в рамках QIM, где один коэффициент модифицируется дважды для встраивания двух битов.

В основе алгоритма, представленного в настоящей статье, лежит формула, предложенная в статье [18]. Исходная формула описывает встраивание информации в пиксели изображения. В настоящей работе она модифицирована для применения к коэффициентам ДКП несжатых цифровых изображений и имеет следующий вид: 


$$
C_{x, y}^{\prime}=\left\{\begin{array}{c}
\operatorname{sgn} C_{x, y}\left(\left[\frac{\left|C_{x, y}\right|}{q}\right]+\left\{\frac{\left|C_{x, y}\right|}{q / 2}\right\}\right), \text { если } b_{1}=0 ; \\
\operatorname{sgn} C_{x, y}\left(\left[\frac{\left|C_{x, y}\right|}{q}\right]+\left\{\frac{\left|C_{x, y}\right|}{q / 2}\right\}+\frac{q}{2}\right), \text { если } b_{1}=1 ;(1)
\end{array}\right.
$$

где $C_{x, y}$ - коэффициент ДКП до встраивания; $C_{x, y}^{\prime}$ коэффициент ДКП после встраивания; $q$ - шаг квантования; $b_{i}$ - бит сообщения; $[a]$ - целая часть $a ;\left\{\frac{a}{b}\right\}$ - остаток от деления $a$ на $b$.

Извлечение информации производится по формуле

$$
b_{i}= \begin{cases}0, & \text { если }\left\{\frac{\left|C_{x, y}^{\prime}\right|}{q}\right\}<\frac{q}{2}, \\ 1, & \text { если }\left\{\frac{\left|C_{x, y}^{\prime}\right|}{q}\right\} \geq \frac{q}{2} .\end{cases}
$$

Алгоритм, основанный на формулах (1) и (2), сформулирован в статье [11]. Его основной особенностью является безошибочное извлечение информации из стегоизображения за счёт итеративной процедуры встраивания, впервые предложенной авторами настоящей работы в статье [19]. Итеративная процедура встраивания заключается в следующем. На этапе встраивания выполняется извлечение информации с проверкой на наличие ошибок. В случае если при извлечении возникли ошибки, они исправляются, а затем выполняется повторное встраивание информации в уже изменённый блок. Если безошибочного извлечения не удалось достичь за определённое число шагов, блок обрабатывается таким образом, чтобы при извлечении ни один из его элементов не мог быть интерпретирован как содержащий встроенный бит. В данном алгоритме для этих целей применяется встраивание бита-флага в один из заранее выбранных коэффициентов. Областью встраивания является 21 высокочастотный коэффициент блока (с номерами 43-63 при зигзаг-сканировании). Эксперименты, проведенные с данным алгорит-
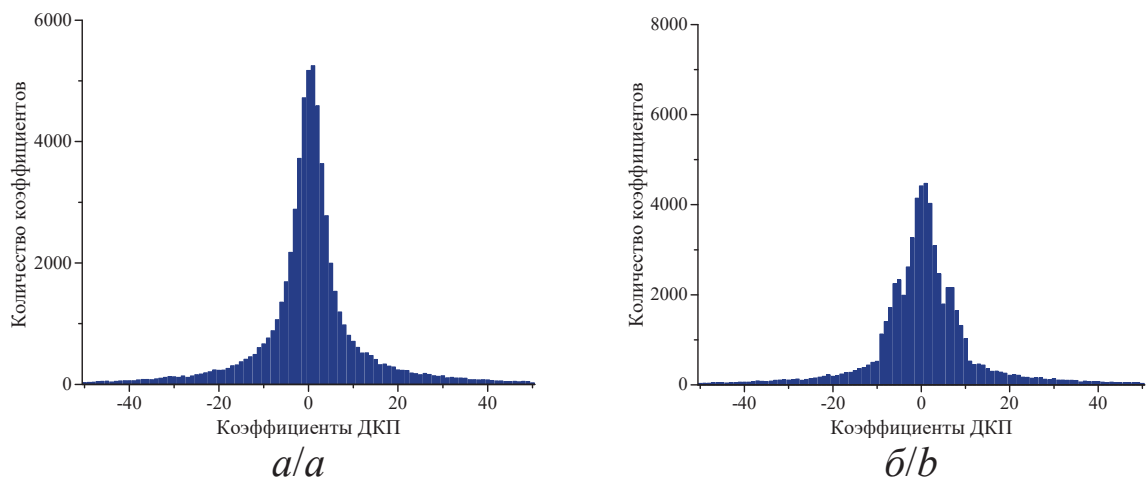

$\sigma / b$ мом, демонстрируют хорошие показатели ёмкости и визуального качества встраивания, оцениваемого по метрике PSNR.

\section{Искажение гистограммы изображения} в области ДКП при встраивании по методу QIM

Несмотря на высокое качество встраивания алгоритм [11] приводит к существенному искажению естественной модели цифрового изображения в частотной области.

Для иллюстрации этой проблемы обратимся $\kappa$ рис. 1 , на котором изображены фрагменты гистограмм распределения значений коэффициентов ДКП до и после встраивания информации согласно указанному алгоритму (шаг квантования $q=10$ ). Вид гистограммы стегоизображения значительно отличается от вида гистограммы оригинального изображения. На гистограмме из рис. 1 , б отчётливо заметно возникновение пиков в районе \pm 5 , что соответствует величине $\pm q / 2$.

Эта проблема [20] является типичной для классического алгоритма QIM. Она связана с тем, что QIM сужает число возможных вариантов значений элементов данных цифрового изображения (пикселей или коэффициентов ДКП), в которые производится встраивание сообщения. В случае конкретного алгоритма возникновение данной проблемы напрямую следует из формулы (1). Например, при встраивании единичного бита в коэффициенты, значения которых близки к нулю, формула преобразуется к виду $\operatorname{sgn} C_{x, y}\left(\left|c_{x, y}\right|+\frac{q}{2}\right)$, что в итоге трансформирует коэффициенты ДКП в значения, близкие к $\pm q / 2$.

Существенное искажение естественной модели цифрового изображения в частотной области напрямую связано с незаметностью встраивания, которое обеспечивает практическую применимость алгоритма сокрытия данных в цифровом объекте.

\section{Восстановление естественной модели цифрового} изображения за счет переменного шага квантования

Чтобы решить указанную проблему, алгоритм [11] был модифицирован таким образом, чтобы шаг квантования для каждого блока коэффициентов ДКП зависел от характеристик этого блока.

Pис.1. Гистограммы изображения: а) до встраивания; б) после встраивания

Fig. 1. Image histograms: a) before embedding; b) after embedding 


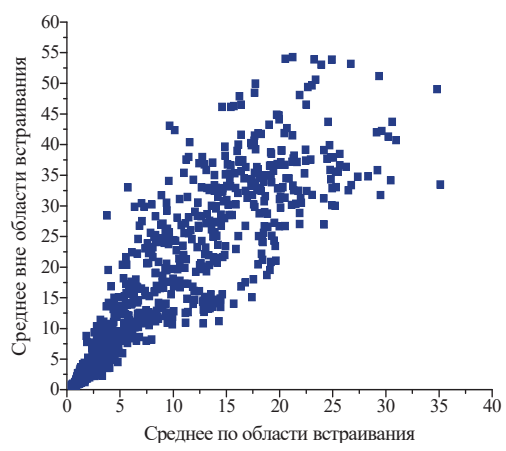

a/a

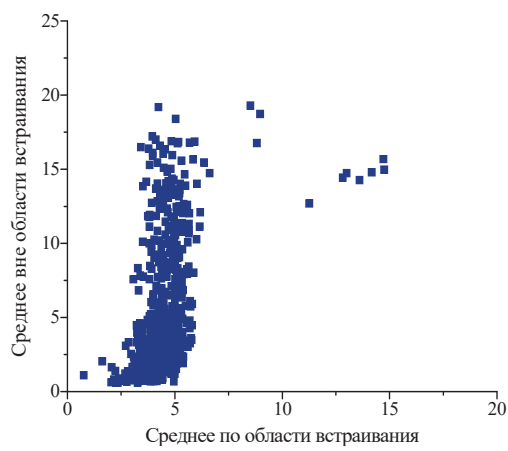

$\sigma / b$

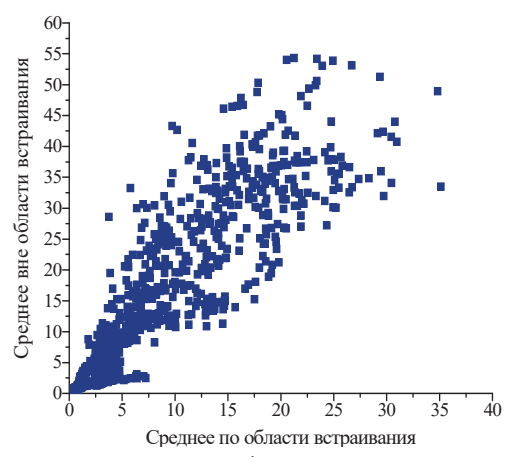

$B / c$

Pис. 2. Соотношение между средними значенияли коэффициентов ДКП для: а) изображения-контейнера; б) стегоизображения, полученного по алгоритму [11]; в) стегоизображения, полученного по улучшенному алгоритлу [11] с переменныл шагом квантования

Fig. 2. Ratio between the average values of DCT coefficients for: a) cover image; b) stego image obtained by the algorithm [11]; c) stego image obtained by the improved algorithm [11] with a variable quantization step

Для этого была введена формула, определяющая величину шага квантования:

$$
q_{n}=\left\{\begin{array}{l}
{[F / E] \cdot K, \text { если } F \geq E,} \\
{[E / F] \cdot K, \text { если } F<E .}
\end{array}\right.
$$

где $E$ - среднее значение модулей $\mathrm{AC}$-коэффициентов ДКП по области встраивания; $F$ - среднее значение модулей AC-коэффициентов ДКП вне области встраивания; $K$ - целочисленный коэффициент; $n$ - номер блока коэффициентов ДКП изображения-контейнера.

Идея подобного способа вычисления шага квантования возникла из следующего наблюдения: соотношение между средними значениями коэффициентов одномерного ДКП по области встраивания и вне её имеет схожий характер для разных изображений. На рис. $2, a$ представлен пример, иллюстрирующий данное соотношение для другого тестового изображения. График на рис. 2, б существенно отличается от графика на рис. $2, a$ по причине искажения естественной модели цифрового изображения в частотной области за счёт встраивания информации с постоянным шагом квантования (шаг квантования $q=10$ ). Однако график, представленный на рис. 2, в, демонстрирует, что встраивание информации с переменным шагом квантования, рассчитанным по формуле (3), позволяет значительно уменьшить изменение данного соотношения.

Рассмотрим влияние произведённой модификации алгоритма на распределение значений коэффициентов ДКП после встраивания. Гистограмма на рис. 3 , б содержит явные признаки наличия вложения. Рис. 3, в демонстрирует влияние переменного шага квантования на степень искажений гистограммы: пики сглажены, гистограмма визуально более похожа на гистограмму исходного изображения.

\section{Результаты экспериментов и обсуждение}

Для проверки эффективности предложенного подхода к корректировке искажений гистограммы была выполнена проверка статистической значимости различий между гистограммой контейнера и стегоизображения с помощью непараметрического критерия Уилкоксона. В ходе экспериментов использовались 20 тестовых изображений из базы USC-SIPI [21], примеры тестовых изображений представлены на рис. 4 . Встраивание произво-
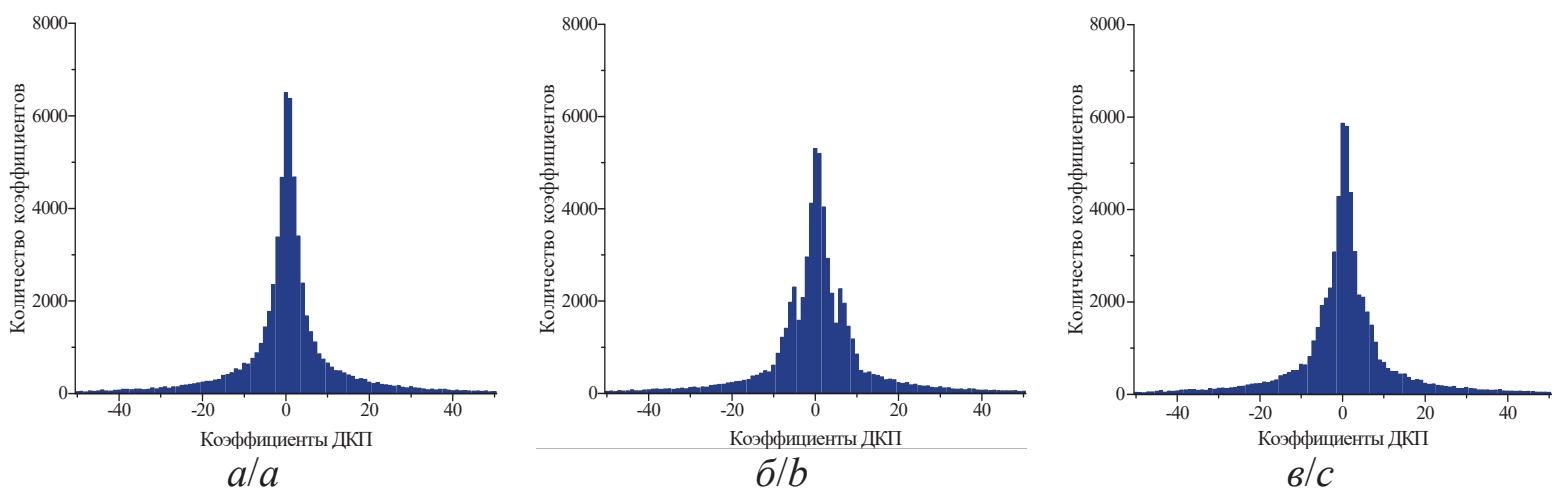

Рис. 3. Гистограмлы изображения: а) до встраивания; б) после встраивания с постоянным шагом; в) после встраивания с переменным шагом

Fig. 3. Image histograms: a) before embedding; $b$ ) after embedding with a constant step; c) after embedding with variable step 

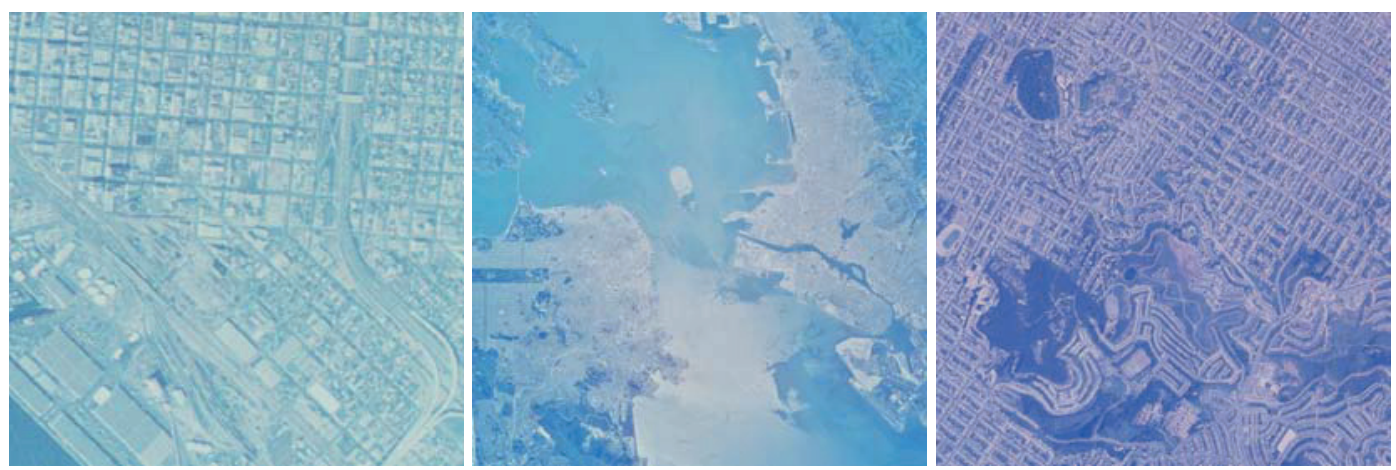

Pис. 4. Примеры тестовых изображений

Fig. 4. Test image examples

дилось только в яркостную составляющую данных изображений, поэтому перед началом экспериментов они были преобразованы в полутоновый вид.

В таблице представлены значения $p$-уровня значимости для данного критерия. Чтобы две гистограммы считались статистически неразличимыми, должно выполняться условие $p>0,05$, в таблице такие значения отмечены серым цветом.

таблица. Проверка статистической различимости гистограмм по критерию Уилкоксона

Table. Checking the statistical legibility of histograms by the Wilcoxon test

\begin{tabular}{|c|c|c|}
\hline \multirow[b]{2}{*}{$\begin{array}{l}\text { Номер изображения } \\
\text { Image number }\end{array}$} & \multicolumn{2}{|c|}{$p$-уровень значимости $/ p$-value } \\
\hline & $\begin{array}{c}\text { Постоянное } \\
\text { Constant } q=5\end{array}$ & $\begin{array}{c}\text { Переменное } \\
\text { Variable } q\end{array}$ \\
\hline 1 & $2,65 \cdot 10^{-5}$ & 0,099 \\
\hline 2 & $6,21 \cdot 10^{-6}$ & 0,663 \\
\hline 3 & $2,03 \cdot 10^{-4}$ & 0,042 \\
\hline 4 & $1,68 \cdot 10^{-7}$ & 0,003 \\
\hline 5 & $2,75 \cdot 10^{-10}$ & 0,053 \\
\hline 6 & $2,06 \cdot 10^{-5}$ & 0,237 \\
\hline 7 & $1,46 \cdot 10^{-6}$ & 0,282 \\
\hline 8 & $1,87 \cdot 10^{-9}$ & 0,105 \\
\hline 9 & $6,41 \cdot 10^{-6}$ & 0,183 \\
\hline 10 & $3,63 \cdot 10^{-5}$ & 0,512 \\
\hline 11 & $2,54 \cdot 10^{-8}$ & 0,624 \\
\hline 12 & $3,90 \cdot 10^{-5}$ & 0,178 \\
\hline 13 & $3,83 \cdot 10^{-10}$ & 0,022 \\
\hline 14 & $1,57 \cdot 10^{-8}$ & 0,060 \\
\hline 15 & $1,37 \cdot 10^{-5}$ & 0,463 \\
\hline 16 & $1,50 \cdot 10^{-4}$ & 0,762 \\
\hline 17 & $2,67 \cdot 10^{-7}$ & 0,414 \\
\hline 18 & $1,63 \cdot 10^{-5}$ & 0,008 \\
\hline 19 & $1,23 \cdot 10^{-10}$ & 0,000 \\
\hline 20 & $9,63 \cdot 10^{-5}$ & 0,550 \\
\hline
\end{tabular}

Как видно из таблицы, при встраивании информации с постоянным шагом квантования гистограммы статистически различимы во всех случаях. Для переменного шага квантования гистограммы до и после встраивания информации статистически неразличимы в $75 \%$ случаев. Данный результат наглядно демонстрирует положительное влияние предложенного подхода на уменьшение искажений естественной модели цифрового изображения в ча- стотной области. При этом качество встраивания улучшилось: при ёмкости 0,11 бит/пиксель среднее значение PSNR составило 40,7 и 41,8 дБ для алгоритмов с постоянным и переменным шагом квантования соответственно.

\section{Заключение}

Решение задачи встраивания информации в снимки дистанционного зондирования Земли необходимо искать в совокупности с решением проблемы обеспечения незаметности встраивания, поскольку серьёзные искажения, внесённые в изображение во время внедрения дополнительных данных, могут негативно сказаться на результатах дальнейшего анализа полученных снимков. В настоящей работе был предложен новый подход к минимизации искажений естественной модели цифрового изображения в области дискретного косинусного преобразования при встраивании информации в снимки дистанционного зондирования Земли по методу QIM, основанный на изменении шага квантования в зависимости от локальных характеристик изображения в частотной области. С помощью данного подхода разработан улучшенный алгоритм встраивания информации в область дискретного косинусного преобразования, который обеспечивает не только безошибочное извлечение информации, но и повышение до $75 \%$ статистической неразличимости изображений-контейнеров и стегоизображений. Наличие эффекта от применения предложенного подхода подтверждается результатами проведённых экспериментов. Полученный алгоритм может эффективно применяться для внедрения дополнительной информации в снимки дистанционного зондирования Земли, при этом позволяя избегать возникновения артефактов встраивания, негативно влияющих на дальнейший анализ таких снимков.

Данная работа выполнена при поддержке Министерства образования и науки Российской Федерации в рамках проектной части государственного задания федерального государственного бюджетного образовательного учреждения высшего образования «Томский государственный университет систем управления и радиоэлектроники» на 2017-2019 г2. (проект № 2.3583.2017/4.6). 


\section{СПИСОК ЛИТЕРАТУРЫ}

1. Семантическая сегментация данных дистанционного зондирования Земли при помощи нейросетевых алгоритмов / А.А. Друки, В.Г. Спицын, Ю.А. Болотова, А.А. Башлыков // Известия Томского политехнического университета. Инжиниринг георесурсов. - 2018. - Т. 329. - № 1. - С. 59-68.

2. Method based on edge constraint and fast marching for road centerline extraction from very high-resolution remote sensing images / L. Gao, W. Shi, Z. Miao, Z. Lv // Remote Sensing. - 2018. V. 10. - № 6. - article number 900.

3. Казарян М.Л., Рихтер А.А., Шахраманьян М.А. Методика автоматического детектирования компонент объектов захоронения отходов по космическим изображениям // Известия Томского политехнического университета. Инжиниринг георесурсов. - 2018. - Т. 328. - № 3. - С. 46-53.

4. Buchnev A.A., Pyatkin V.P., Rusin E.V. Software Technologies for Processing of Earth Remote Sensing Data // Pattern Recognition and Image Analysis. - 2013. - V. 23. - № 4. - P. 474-480.

5. Serra-Ruiz J., Megías D. A novel semi-fragile forensic watermarking scheme for remote-sensing images // International Journal of Remote Sensing. - 2011. - V. 32. - № 19. - P. 5583-5606.

6. Serra-Ruiz J., Megías D. Reversible data hiding for tampering detection in remote sensing images using histogram shifting // Proceedings of SPIE - The International Society for Optical Engineering. - 2012. - V. 8514. - 85140Y.

7. Митекин В.А. Алгоритм генерации стойкого цифрового водяного знака для защиты гиперспектральных изображений дистанционного зондирования Земли // Компьютерная оптика. 2015. - T. 39. - № 5. - C. 808-817.

8. Khosravi M.R., Rostami H., Samadi S. Enhancing the binary watermark-based data hiding scheme using an interpolation-based approach for optical remote sensing images // International Journal of Agricultural and Environmental Information Systems. 2018. - V. 9. - № 2. - P. 53-71.

9. Akhtarkavan E., Majidi B., Manzuri M.T. Secure communication and archiving of low altitude remote sensing data using high capacity fragile data hiding // Multimedia Tools and Applications. - 2018. - P. 1-27.

10. One-pass lossless data hiding and compression of remote sensing data / B. Carpentieri, A. Castiglione, A. De Santis, F. Palmieri, R. Pizzolante // Future Generation Computer Systems. - 2019. V. 90. - P. 222-239.

11. Евсютин 0.0., Кокурина А.С., Мещеряков Р.В. Алгоритм встраивания информации в цифровые изображения с безошибочным извлечением, основанный на методе QIM // Распреде- ленные информационно - вычислительные ресурсы. Наука цифровой экономике: Труды XVI Всероссийской конференции. - Россия, Новосибирск, 2017. - С. 314-322.

12. Chen B., Wornell G.W. Quantization index modulation: a class of provably good methods for digital watermarking and information embedding // IEEE Transactions on Information Theory. 2001. - V. 47. - № 4. - P. 1423-1443.

13. Borah S., Borah B. Quantization index modulation (QIM) based watermarking techniques for 3D meshes // Proceedings of the Fourth International Conference on Image Information Processing (ICIIP). - India, Shimla, 2017. - P. 1-6.

14. Zhu X., Tang Z. Improved quantization index modulation watermarking robust against amplitude scaling and constant change distortions // Proceedings of the $15^{\text {th }}$ IEEE International Conference on Image Processing. - USA, San Diego, 2008. P. 433-436.

15. Noda H., Niimi M., Kawaguchi E. Application of QIM with dead zone for histogram preserving JPEG steganography // Proceedings of the IEEE International Conference on Image Processing. - Italy, Genova, 2005. - V. 2. - P. 1082-1085.

16. Noda H., Tsukamizu Y., Niimi M. JPEG2000 steganography possibly secure against histogram-based attack / // Proceedings of the $7^{\text {th }}$ Pacific Rim Conference on Multimedia. - China, Hangzhou, 2006. - P. 80-87.

17. Sarkar A., Manjunath B.S. Double embedding in the quantization index modulation framework // Proceedings of the $16^{\text {th }}$ IEEE International Conference on Image Processing (ICIP). - Egypt, Cairo, 2009. - P. 3653-3656.

18. Глумов Н.И., Митекин В.А. Алгоритм встраивания полухрупких цифровых водяных знаков для задач аутентификации изображений и скрытой передачи информации // Компьютерная оптика. - 2011. - Т. 35. - № 2. - C. 262-267.

19. An adaptive algorithm for the steganographic embedding information into the discrete Fourier transform phase spectrum / 0.0. Evsutin, A.S. Kokurina, R.V. Mescheryakov, 0.0. Shumskaya // Intelligent Information Technologies for Industry: Proceedings of the First International Scientific Conference. - Russia, Sochi, 2016. - P. 47-56.

20. Mitekin V., Fedoseev V. A new QIM-based watermarking algorithm robust against multi-image histogram attack // Procedia Engineering. - 2017. - V. 201. - P. 453-462.

21. The USC-SIPI image database. URL: http://sipi.usc.edu/database/ (дата обращения: 24.11.2018).

Поступила 31.05.2019 2.

\section{Информация об авторах}

Eвсютин 0.O., кандидат технических наук, доцент кафедры безопасности информационных систем Томского государственного университета систем управления и радиоэлектроники.

Кокурина A.C., младший научный сотрудник кафедры безопасности информационных систем Томского государственного университета систем управления и радиоэлектроники.

Мещеряков P.B., доктор технических наук, главный научный сотрудник Института проблем управления им. В.А. Трапезникова Российской академии наук. 
UDC 004.021

\title{
STEGANOGRAPHIC EMBEDDING OF ADDITIONAL DATA INTO THE IMAGES OF EARTH REMOTE SENSING BY QIM METHOD WITH A VARIABLE QUANTIZATION STEP IN THE FREQUENCY DOMAIN
}

Oleg 0. Evsyutin',

eoo@keva.tusur.ru

\author{
Anna S. Kokurina', \\ annakokurina94@yandex.ru \\ Roman V. Meshcheryakov², \\ mrv@ipu.ru \\ 1 'Tomsk State University of Control Systems and Radioelectronics, \\ 40, Lenin Avenue, Tomsk, 634050, Russia. \\ 2 V.A. Trapeznikov Institute of Control Sciences of Russian Academy of Sciences, \\ 65, Profsoyuznaya street, Moscow, 117997, Russia.
}

\begin{abstract}
The relevance. One of the areas of digital image processing, including images of Earth remote sensing, is associated with the embedding of additional information into such images for various purposes. Embedding of additional information into a digital image leads to distortion of the digital image natural model, as well as to the possible occurrence of visual artifacts. In the case of images of Earth remote sensing, such distortions may entail, for example, distortion of the object boundaries, as a result of which further analysis of the images will lead to incorrect results. Therefore, the studies aimed at finding new ways to reduce distortions caused by embedding additional information are relevant.

The aim of the research is to improve the quality of steganographic embedding of information in the coefficients of discrete cosine transform of Earth remote sensing images by developing an improved embedding algorithm based on the QIM method and ensuring the correction of distortions of the natural image model in the frequency domain.

Objects: algorithms for steganographic embedding of information in the coefficients of digital images discrete cosine transform.

Methods: QIM steganographic method, methods of mathematical statistics, computational experiments.

Results. The paper proposes a new approach to minimizing the distortion of the natural model of a digital image in the discrete cosine transform domain, based on changing the quantization step depending on the local image characteristics in the frequency domain. The result of the work is an improved algorithm for embedding the information in the coefficients of discrete cosine transform of images of Earth remote sensing, combining this approach with an approach to ensure the correct extraction of embedded information proposed by the authors earlier. The results of the experiments show that the developed algorithm, along with ensuring the accuracy of extraction, can significantly reduce the distortions introduced into frequency coefficients and ensure statistical indistinguishability of the cover images and stego images in $75 \%$ of cases.
\end{abstract}

Key words:

Digital images, steganography, discrete cosine transform, QIM, Earth remote sensing.

The work was funded by the Russian Federation Ministry of Education and Science (grant 2.3583.2017/4.6).

\section{REFERENCES}

1. Druki A.A., Spitsyn V.G., Bolotova Yu.A., Bashlykov A.A. Semantic segmentation of Earth scanning images using neural network algorithms. Bulletin of the Tomsk Polytechnic University. Geo Assets Engineering, 2018, vol. 329, no. 1, pp. 59-68. In Rus.

2. Gao L., Shi W., Miao Z., Lv Z. Method based on edge constraint and fast marching for road centerline extraction from very highresolution remote sensing images. Remote Sensing, 2018, vol. 10, no. 6 , article number 900 .

3. Kazaryan M.L., Richter A.A., Shakhramanian M.A. Method of automatic detecting the components of waste disposal objects by space images. Bulletin of the Tomsk Polytechnic University. Geo Assets Engineering, 2018, vol. 328, no. 3, pp. 46-53. In Rus.

4. Buchnev A.A., Pyatkin V.P., Rusin E.V. Software Technologies for Processing of Earth Remote Sensing Data. Pattern Recognition and Image Analysis, 2013, vol. 23, no. 4, pp. 474-480.

5. Serra-Ruiz J., Megías D. A novel semi-fragile forensic watermarking scheme for remote-sensing images. International Journal of Remote Sensing, 2011, vol. 32, no 19, pp. 5583-5606.

6. Serra-Ruiz J., Megías D. Reversible data hiding for tampering detection in remote sensing images using histogram shifting. Proce- edings of SPIE - The International Society for Optical Engineering, 2012, vol. 8514, article number 85140Y.

7. Mitekin V.A. An algorithm for generating digital watermarks robust against brute-force attacks. Computer Optics, 2015, vol. 39, no. 5, pp. 808-817. In Rus.

8. Khosravi M.R., Rostami H., Samadi S. Enhancing the binary watermark-based data hiding scheme using an interpolation-based approach for optical remote sensing images. International Journal of Agricultural and Environmental Information Systems, 2018, vol. 9, no. 2, pp. 53-71.

9. Akhtarkavan E., Majidi B., Manzuri M.T. Secure communication and archiving of low altitude remote sensing data using high capacity fragile data hiding. Multimedia Tools and Applications, 2018, pp. 1-27.

10. Carpentieri B., Castiglione A., De Santis A., Palmieri F., Pizzolante R. One-pass lossless data hiding and compression of remote sensing data. Future Generation Computer Systems, 2019, vol. 90 , pp. 222-239.

11. Evsutin 0.0., Kokurina A.S., Meshcheryakov R.V. Algorithm of the Information Embedding in Digital Images with Unmistakable Extraction Based on QIM Method. Proceedings of the XVI All-Rus- 
sian Conference. Distributed Information and Computing Resources. Science to the digital economy (DICR-2017). Russia, Novosibirsk, 2017. pp. 314-322. In Rus.

12. Chen B., Wornell G.W. Quantization index modulation: a class of provably good methods for digital watermarking and information embedding. IEEE Transactions on Information Theory, 2001, vol. 47, no. 4, pp. 1423-1443.

13. Borah S., Borah B. Quantization index modulation (QIM) based watermarking techniques for 3D meshes. Proceedings of the Fourth International Conference on Image Information Processing (ICIIP). India, Shimla, 2017. pp. 1-6.

14. Zhu X., Tang Z. Improved quantization index modulation watermarking robust against amplitude scaling and constant change distortions. Proceedings of the $15^{\text {th }}$ IEEE International Conference on Image Processing. USA, San Diego, 2008. pp. 433-436.

15. Noda H., Niimi M., Kawaguchi E. Application of QIM with dead zone for histogram preserving JPEG steganography. Proceedings of the IEEE International Conference on Image Processing. Italy, Genova, 2005. Vol. 2, pp. 1082-1085.

16. Noda H., Tsukamizu Y, Niimi M. JPEG2000 steganography possibly secure against histogram-based attack. Proceedings of the $7^{\text {th }}$ Pacific Rim Conference on Multimedia. China, Hangzhou, 2006. pp. 80-87.
17. Sarkar A., Manjunath B.S. Double embedding in the quantization index modulation framework. Proceedings of the $16^{\text {th }}$ IEEE International Conference on Image Processing (ICIP). Egypt, Cairo, 2009. pp. 3653-3656.

18. Glumov N.I., Mitekin V.A. A new semi-fragile watermarking algorithm for image authentication and information hiding. Computer Optics, 2011, vol. 35, no. 2, pp. 262-267. In Rus.

19. Evsutin 0., Kokurina A., Mescheryakov R., Shumskaya 0. An adaptive algorithm for the steganographic embedding information into the discrete Fourier transform phase spectrum. Proceedings of the First International Scientific Conference. Intelligent Information Technologies for Industry (IITI'16). Russia, Sochi, 2016. pp. 47-56.

20. Mitekin V., Fedoseev V. A new QIM-based watermarking algorithm robust against multi-image histogram attack. Procedia Engineering, 2017, vol. 201, pp. 453-462.

21. The USC-SIPI image database. Available at: http://sipi.usc.edu/ database/ (accessed: 24 November 2018).

Received: 31 May 2019.

\section{Information about the authors}

Oleg O. Evsyutin, Cand. Sc., associate professor, Tomsk State University of Control Systems and Radioelectronics.

Anna S. Kokurina, junior researcher, Tomsk State University of Control Systems and Radioelectronics.

Roman V. Meshcheryakov, Dr. Sc., chief researcher, V.A. Trapeznikov Institute of Control Sciences of Russian Academy of Sciences. 